\begin{tabular}{|c|c|c|c|c|c|c|}
\hline \multirow{4}{*}{ Impact Factor: } & ISRA (India) & $=3.117$ & SIS (USA) & $=0.912$ & ICV (Poland) & $=6.630$ \\
\hline & ISI (Dubai, UAE & $=0.829$ & РИНЦ (Russia & $=0.156$ & PIF (India) & $=1.940$ \\
\hline & GIF (Australia) & $=0.564$ & ESJI (KZ) & $=8.716$ & IBI (India) & $=4.260$ \\
\hline & JIF & $=1.500$ & SJIF (Morocco & $=5.667$ & OAJI (USA) & $=0.350$ \\
\hline
\end{tabular}

\section{SOI: $1.1 /$ TAS $\quad$ DOI: $10.15863 /$ TAS \\ International Scientific Journal Theoretical \& Applied Science}

\author{
p-ISSN: 2308-4944 (print) e-ISSN: 2409-0085 (online) \\ Year: 2019 Issue: 08 Volume: 76
}

Published: 05.08.2019 http://T-Science.org
QR - Issue

QR - Article

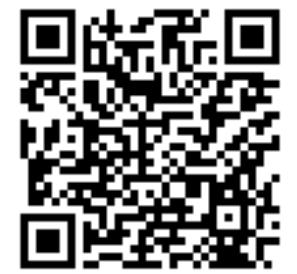

Farah Polad Rahmani Azerbaijan State Economic University dissertant

Baku, Azerbaijan Republic

\title{
THE PROBLEMS AND PROSPECTS OF IMPROVING THE ORGANIZATIONAL AND ECONOMIC MECHANISMS OF INNOVATIVE DEVELOPMENT OF INDUSTRIAL ENTERPRISES
}

\begin{abstract}
The problems and prospects of improving the organizational and economic mechanisms of innovative development of industrial enterprises are explored in the article. The essence of the concepts of "innovation" and "innovative processes", the main factors affecting the main organizational and economic mechanisms of innovative development of industrial enterprises is disclosed. The advantage of innovation and its impact on increasing labor productivity, a list of competitive and export-oriented innovative products is considered. The importance of a systematic approach to the organization and development of innovative activities in enterprises, the strengthening of research and development, the development, development and application of new innovations with the further provision of the production of innovative products is noted. The state of the ongoing work on the formation and development of innovative activity in Azerbaijan, where innovative activity, including the innovative development of industrial enterprises, is at the initial stage is assessed. The importance of a comprehensive solution to the problems that hinder the intensification of the innovative development of industrial enterprises is noted. A number of recommendations and suggestions on problems and prospects for improving the organizational and economic mechanisms for the innovative development of industrial enterprises in modern conditions and in the near future are prepared.

Key words: innovation, innovative processes, innovative strategy of an enterprise, innovative development of an enterprise, organizational and economic mechanisms for the innovative development of industrial enterprises, innovative system in Azerbaijan, innovative development of industrial enterprises in Azerbaijan.

Language: Russian

Citation: Rahmani, F. P. (2019). The problems and prospects of improving the organizational and economic mechanisms of innovative development of industrial enterprises. ISJ Theoretical \& Applied Science, 08 (76), 11-16.

Soi: http://s-o-i.org/1.1/TAS-08-76-3 Doi: crossef https://dx.doi.org/10.15863/TAS.2019.08.76.3

Classifiers: Economic research, finance, innovation, risk management.

\section{ПРОБЛЕМЫ И ПЕРСПЕКТИВЫ СОВЕРШЕНСТВОВАНИЯ ОРГАНИЗАЦИОННО- ЭКОНОМИЧЕСКИХ МЕХАНИЗМОВ ИННОВАЦИОННОГО РАЗВИТИЯ ПРОМЫШЛЕННЫХ ПРЕДПРИЯТИЙ}

Аннотация: В статье исследованы проблемы и перспективы совершенствования организационноэкономических механизмов инновационного развития промышленных предприятий. Раскрыта сущность понятий «инновация» $u$ «инновационные процессы», основных факторов, влияющих на главные организационно-экономические механизмы инновационного развития промышленных предприятий. Рассмотрено преимущество инновационной деятельности и его влияние на повышение производтельности труда, перечня конкурентоспособных и экспортно-ориентированных инновационных продукций. Отмечена важность системного подхода к организации и развитию инновационной деятельности в предприятиях, усилению научно-исследовательской работы, разработкам, освоению и применению новых инноваций с дальнейшим обеспечением производства инновационных продукций. Оченено состояние проводимых работ по формированию и развитию инновационной деятельности в Азербайджане, где инновационная 


\begin{tabular}{llllll} 
& ISRA (India) $=3.117$ & SIS (USA) $=\mathbf{0 . 9 1 2}$ & ICV (Poland) & $=\mathbf{6 . 6 3 0}$ \\
Impact Factor: & ISI (Dubai, UAE) $=\mathbf{0 . 8 2 9}$ & PUHЦ (Russia) $=\mathbf{0 . 1 5 6}$ & PIF (India) & $=\mathbf{1 . 9 4 0}$ \\
& GIF (Australia) $=\mathbf{0 . 5 6 4}$ & ESJI (KZ) & $\mathbf{8 . 7 1 6}$ & IBI (India) & $=\mathbf{4 . 2 6 0}$ \\
& JIF & $=\mathbf{1 . 5 0 0}$ & SJIF (Morocco) $=\mathbf{5 . 6 6 7}$ & OAJI (USA) & $\mathbf{0 . 3 5 0}$ \\
\hline
\end{tabular}

деятельность, в том числе инновационное развитие промышленных предприятий находится на начальной стадии. Отмечена важность комплексного решения проблем, мешающих интенсификации инновационного развития промышленных предприятий. Подготовлен ряд рекомендаций и предложений по проблемам и перспективам совершенствования организачионно-экономических механизмов инновационного развития промышленных предприятий в современных условиях и ближайшей перспективе.

Ключевые слова: инновачия, инновационные прочессы, инновачионная стратегия предприятия, инновационное развитие предприятия, организационно-экономические механизмы инновационного развития промышленных предприятий, инновационная система в Азербайджане, инновационное развитие промышленных предприятий Азербайджана.

\section{Введение}

Проблемы совершенствования экономических механизмов инновационного развития промышленных предприятий с каждым годом становятся одними из решающих компонентов и приоритетов по модернизации основных механизмов национальной экономики, прежде всего, его промышленного сектора, формирования новых продуктивных источников роста и создания добавочной стоимости, расширения перечня конкурентоспособных высокотехнологичных инноционных продукций, повышение экспортного потенциала и в целом обеспечение стабильности функционирования отдельных секторов экономики страны в условиях углубления глобальных угроз и воздействий.

Как известно, термин «инновация» произошел от латинского слова «innovation», которое означает «обновление», «улучшение» и имеет схожее значение со словами «новшество» и «нововведение». Инновация рассматривается как развивающийся комплексный процесс создания и распространения. Й.Шумпетер трактует инновацию как новую комбинацию производственных факторов, мотивированную предпринимательским духом, а инновационные процессы - происходящие в результате создания нового продукта и его освоения процессы [1]. По мнению О.Б.Трофимова «под инновацией мы будем понимать результат создания востребованных обществом различных новшеств, являющихся более совершенными, чем их аналоги, в результате использования которых обеспечивается достижение прибыли предприятий [2, с.23]. Инновации выступают основой для обновления и придания нового импульса в экономических и хозяйственных процессах, позволяет разработке и осуществлению новых механизмов и инструментарий, в целом система инноваций характеризуется в качестве совокупности процессов создания и расширения среды инновационного развития, где больше всего присутствуют новые подходы, новые товары и продукции, которые в корне отличаются от своих предыдущих аналогов. Основным определяющим условием формирования и распространения новых инноваций, организации инновационной деятельности предприятия является получение результата деятельности по созданию нового продукта, услуги, процесса [3]. Инновационные процессы способствуют ускорению рациональности предпринимательской деятельности и бизнес-процессов. В результате применения инновационной функции производительность и конкурентоспособность предпринимательской деятельности существенно повышается. По мнению П.Ф.Друкера, на предприятиях к двум важным функциям менеджера должны относиться - внедрение инноваций и осуществление маркетинговой деятельности, при этом необходимо полагаться на технологии и дать предпочтение внедрению инноваций [4, с.220]. Кроме того, отметим, что инновационная стратегия и инновационная деятельность в целом, инновационное развитие промышленных предприятий обуславливает учет всех основных элементов ведения хозяйственной деятельности для того, чтобы в конечном итоге предприятие добилось прогнозируемых результатов с обязательным условием обеспечения прибыльности инновационной деятельности, повышения его конкурентоспособности. Инновационное развитие, обусловленное развитием производительных сил, объективно присущих каждой национальной экономике, но определенной ступени развития она оформляется в конкретную систему - национальноинновационную систему, действующую по характерным для системы принципам. Стратегической целью данной системы объясняется необходимость создания организационно-экономической инновационной деятельности, обеспечение более продуктивными и эффективными экономическими механизмами инновационной стратегии предприятия [5, с.153]. Как отмечает Г.Ф.Фейгин, в условиях глобальных изменений и трансформаций экономических процессов необходимо изменение основных механизмов мирохозяйственной системы, решение проблем диверсификации и повышение технологического уровня национальной экономики, в первую очередь, в промышленной сфере. В связи с этим одним из важных условий является развитие национальной экономики за 


\begin{tabular}{llllll} 
& ISRA (India) $=3.117$ & SIS (USA) $=\mathbf{0 . 9 1 2}$ & ICV (Poland) & $=\mathbf{6 . 6 3 0}$ \\
Impact Factor: & ISI (Dubai, UAE) $=\mathbf{0 . 8 2 9}$ & PUHЦ (Russia) $=\mathbf{0 . 1 5 6}$ & PIF (India) & $=\mathbf{1 . 9 4 0}$ \\
& GIF (Australia) $=\mathbf{0 . 5 6 4}$ & ESJI (KZ) & $\mathbf{8 . 7 1 6}$ & IBI (India) & $=\mathbf{4 . 2 6 0}$ \\
& JIF & $=\mathbf{1 . 5 0 0}$ & SJIF (Morocco) $=\mathbf{5 . 6 6 7}$ & OAJI (USA) & $\mathbf{0 . 3 5 0}$ \\
\hline
\end{tabular}

счет высоких технологий и инновационных функций [6, с.304]. Инновационные функции способствуют изменению отношения к существующим проблемам и недостаткам, нахождению наиболее оптимальных и эффективных решений, разработке новых механизмов по их устранению и ускорению модернизации устаревшего оборудования, обновлению технологий, активному участию в технологическом обмене и трансфере технологий на международной арене. Инновации помогают успешно регулировать создавшиеся диспропорции в деятельности отдельных сфер промышленного сектора национальной экономики, обеспечить его диверсификацию, рост, повышение объема формирования национального дохода и добавочной стоимости [7, c.213].

Для обеспечения эффективности и продуктивности организационно-экономических механизмов инновационного развития промышленных предприятий необходима более обоснованная и сбалансированная инновационная стратегия, где должны сконцентрироваться основные приоритеты и направления инновационной деятельности, тактика и инструментарии, маркетинговые подходы инновационного развития, вопросы и приоритеты научно-исследовательской деятельности, а также взаимодействие промышленных предприятий с научно-исследовательскими учреждениями и вузами, государственными структурами, инвестиционно-инновационными фондами и субъектами международной инновационноинвестиционной деятельности. Неэффективность стратегии затрудняет обоснованное определение научно-технических приоритетов, механизмов их реализации, совокупных расходов на науку и их структуру, направлений научно-технического развития, а, следовательно, не дает возможность предприятиям выявить четкие ориентиры своего инновационного развития [8, с.3]. Кроме того, для реализации важнейших мероприятий и механизмов инновационной стратегии предприятия требуется более оптимальная и обоснованная разработка и реализация инновационных проектов. Так, справедливо С.А.Варламов отмечает, что для успешной реализации инновационных проектов необходимо в обязательном порядке учитывать ряд стратегических факторов:

1. рентабельность инноваций;

2. новые инновационные продукты, которые отличаются конкурентоспособностью;

3. инновационные идеи, которые получили одобрение специалистов предприятия;

4. повышение рыночной стоимости предприятия;

5. рост выручки от реализации за счет новой инновационной продукции;

6. бенчмаркинг - непрерывный процесс оценки уровня инновационной продукции;

7. время вывода новой инновационной продукции на рынок;

8. использование

конкурентных преимуществ предприятия;

9. доля реализованных инновационных идей и предложений;

10. качество информационного обеспечения организации и др. [9, с.70].

Следует подчеркнуть, что к главным группам инноваций относятся новые инновационные продукции, при этом инновации отличаются многофункциональностью и в дальнейшем происходит разработка, освоение и применение ряда важнейших этапов инновационного развития: технических, производственный и маркетинговый процессы, на этих этапах оптимальное планирование и прогнозирование инновационной деятельности обуславливает последовательную реализацию организационного-экономических механизмов инновационного развития промышленных предприятий.

На Рисунке 1 даны основные факторы инновационной деятельности и их влияние на механизмы инновационного развития промышленных предприятий в нынешних условиях. 


\begin{tabular}{llllll} 
& ISRA (India) $=\mathbf{3 . 1 1 7}$ & SIS (USA) $=\mathbf{0 . 9 1 2}$ & ICV (Poland) & $=\mathbf{6 . 6 3 0}$ \\
Impact Factor: & ISI (Dubai, UAE) $=\mathbf{0 . 8 2 9}$ & PUHЦ (Russia) $=\mathbf{0 . 1 5 6}$ & PIF (India) & $=\mathbf{1 . 9 4 0}$ \\
& GIF (Australia) $=\mathbf{0 . 5 6 4}$ & ESJI (KZ) $=\mathbf{8 . 7 1 6}$ & IBI (India) & $=\mathbf{4 . 2 6 0}$ \\
& JIF & $\mathbf{1 . 5 0 0}$ & SJIF (Morocco) $=\mathbf{5 . 6 6 7}$ & OAJI (USA) & $\mathbf{= 0 . 3 5 0}$ \\
\hline
\end{tabular}
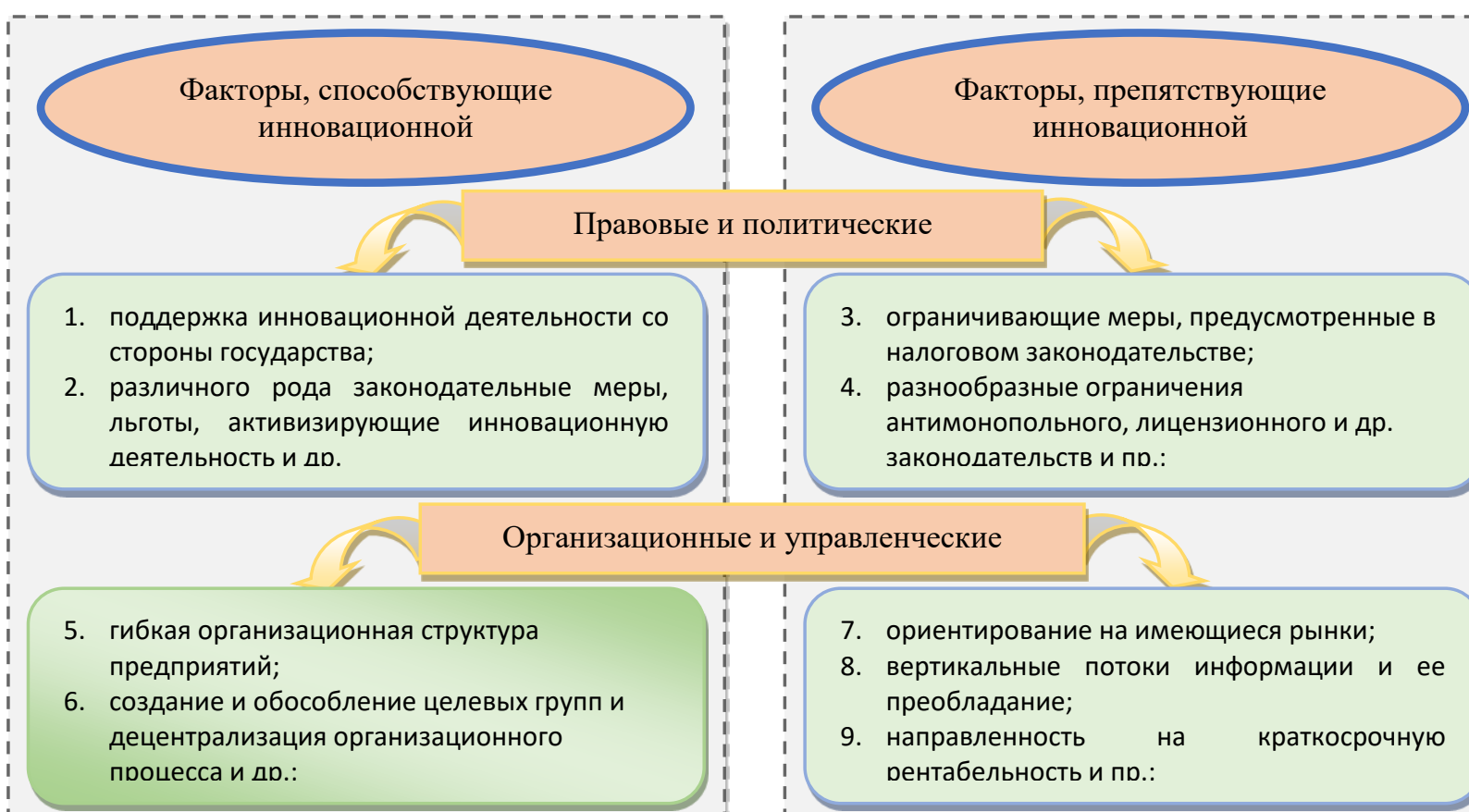

3. ограничивающие меры, предусмотренные в налоговом законодательстве;

4. разнообразные ограничения антимонопольного, лицензионного и др. законодательств и пр.:

10. присутствие соответствующей технической и научной инфраструктуры;

11. система мотиваций и поощрений инновационной деятельности;

12. наличие материально-технической базы и
7. ориентирование на имеющиеся рынки;

8. вертикальные потоки информации и еe преобладание;

9. направленность на краткосрочную рентабельность и пр.:
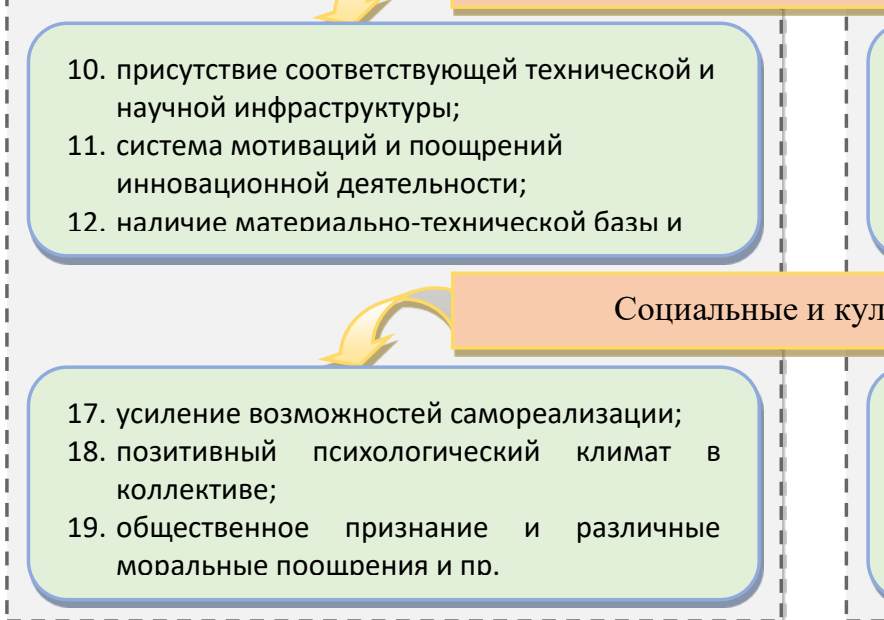

\section{3. преобладание производства;}

14. недостаточное количество финансовых средств для инновационных проектов;

15. отситствие резервных фондов и пр.:

Рисунок 1. Основные факторы инновационной деятельности и их влияние на механизмы инновационного развития промышленных предприятий в нынешних условиях (составлено автором на основе материалов исследования).

Как показывает анализ Рисунка 1, среди основных факторов инновационной деятельности и их влияния на инновационное развитие промышленных предприятий в современных условиях большего всего выделяются: правовые и политические факторы, которые оказывают серьезное влияние на обеспечение инновационного развития; организационные и управленческие факторы, способствующие созданию соответствующих механизмов, развитию инновационной системы; экономические и технологические факторы, которые играют важную роль в обеспечении материально-технической базы и инфраструктуры инновационной деятельности; социальные и
20. опасение неопределенности и убыточности;

21. традиционный образ мышления;

22. сопротивление новизне, изменениям в трудовом процессе и др.

культурные факторы, в конечном результате оказывающие серьезное влияние на процессы сбалансирования инновационного развития экономики и общества, повышение жизненного уровня населения страны. Кроме того, особое значение имеет объективная оценка взаимодействующих факторов, которые серьезно воздействуют на рациональность инновационной деятельности промышленных предприятий. Инновационному менеджменту предприятия необходимо консолидировать все элементы и компоненты потенциального инновационного развития предприятия, мобилизовать интеллектуальные, организационные, человеческие ресурсы, своевременно осуществить 


\begin{tabular}{llllll} 
& ISRA (India) $=\mathbf{3 . 1 1 7}$ & SIS (USA) $=\mathbf{0 . 9 1 2}$ & ICV (Poland) & $\mathbf{= 6 . 6 3 0}$ \\
Impact Factor: & ISI (Dubai, UAE) $=\mathbf{0 . 8 2 9}$ & PUHЦ (Russia) $=\mathbf{0 . 1 5 6}$ & PIF (India) & $=\mathbf{1 . 9 4 0}$ \\
& GIF (Australia) $=\mathbf{0 . 5 6 4}$ & ESJI (KZ) $=\mathbf{8 . 7 1 6}$ & IBI (India) & $=\mathbf{4 . 2 6 0}$ \\
& JIF & $\mathbf{1 . 5 0 0}$ & SJIF (Morocco) $=\mathbf{5 . 6 6 7}$ & OAJI (USA) & $\mathbf{0 . 3 5 0}$ \\
\hline
\end{tabular}

долгосрочное планирование и прогнозирование ресурсного потенциала инновационного развития предприятия согласно инновационной стратегии, осуществить скрупулёзный анализ с соответствующими выводами по каждому звену инновационной цепочки, в том числе по механизму управлению инновационными процессами. Исследователь А.М.Губернаторов в качестве методологических подходов по управлению инновационного развития отрасли выделяет:

1. централизованный - где присутствует и доминирует государственное регулирование, его механизмы и инструменты;

2. функциональный - _ например, маркетинговые, технологические инновации и т.д.;

3. процессный - подразумевает в себе конкретные инновационные бизнес-процессы;

4. ситуационный - усиление контроля за инновационными процессами; 5) динамический взаимодействие инновационной деятельности с участием субъектов отрасли;

5. процессно-синергетический - ставка делается на процессные инновации с учетом синергетического преимущества;

6. проектный - больше всего практикуется в формировании и развитии инновационной деятельности предприятия, в котором предпочтение отдается разработке и реализации отдельных и конкретных инновационных проектов;

7. мезоэкономический - субъектом инновационного развития выступают конкретные экономические субъекты;

8. многомерный - подразумевает многомерность инновационных механизмов, нацеленных на достижение высокотехнологичных результатов и

9. нелинейный - главным компонентом инновационной деятельности является мировоззренческие инновации с учетом современных инновационных вызовов [10, с.5152].

Отметим, что инновационное развитие экономики Азербайджана и, в том числе, промышленных предприятий, за последнее десятилетие набирает обороты, однако пока эти процессы не перешли в интенсивную фазу. Данные аспекты развития отражены в ряде стратегических документов, концепций и государственных программ, в которых намечены комплексные мероприятия, институциональные и инновационные механизмы ускорения инновационного развития в Азербайджане. Так, в концепции развития «Азербайджан - 2020: взгляд в будущее» отмечается, что расширение инновационной деятельности будет одним из основных направлений для обеспечения долгосрочного устойчивого экономического развития, формирования экономики знаний, ускорения создания наукоемких технологий и продуктов $[11$, с.20]. В числе приоритетных направлений инновационной деятельности намечается усиление связи между наукой и производством, усиление государственной поддержки и создание законодательной основы для повышения инновационной активности, обеспечения рационального использования и развития инновационного потенциала в стране. Одним из приоритетных направлений инновационного развития в Азербайджане предусмотрено развитие инновационного предпринимательства и с этой целью происходит активизация трансфера передовых технологий изза границы, создание технопарков и инновационных зон для разработки и применения высокотехнологичной промышленной продукции. В этих направлениях уже имеются первые результаты - в Сумгайытском промышленном центре действует высокотехнологичный парк, а в Пираллахинском промышленном парке идет интенсивная работа по созданию инновационных предприятий. Кроме того, в Государственной Программе по развитию промышленности в Азербайджанской Республике на 2015-2020 годы, утвержденной Распоряжением Президента Азербайджанской Республики от 26 декабря 2014 года отмечается, что одной из стратегических целей развития промышленности страны является расширение наукоемких и инновационных производств, создание промышленных и технологических парков, усиление государственной поддержки с целью расширения инновационной деятельности и стимулирование внедрения инновационных технологий в промышленных предприятиях [12, с.16]. Однако процессы формирования и развития инновационной системы, а также инноватизации промышленных предприятий в Азербайджане не набирали должного уровня.

В целом, таким образом, для решения накопившихся проблем и реальной оценки существующих резервов по раскрытию и реализации перспективы совершенствования организационно-экономических механизмов инновационного развития промышленных предприятий необходимо учитывать ряд принципов и факторов и дальнейшей их последовательной реализации:

- необходимо системно и комплексно подходить к проблемам организационноэкономических механизмов инновационного развития промышленных предприятий и обеспечить их продуктивную реализацию; 


\begin{tabular}{llllll} 
& ISRA (India) $=\mathbf{3 . 1 1 7}$ & SIS (USA) $=\mathbf{0 . 9 1 2}$ & ICV (Poland) & $\mathbf{= 6 . 6 3 0}$ \\
Impact Factor: & ISI (Dubai, UAE) $=\mathbf{0 . 8 2 9}$ & PUHL (Russia) $=\mathbf{0 . 1 5 6}$ & PIF (India) & $=\mathbf{1 . 9 4 0}$ \\
& GIF (Australia) $=\mathbf{0 . 5 6 4}$ & ESJI (KZ) & $=\mathbf{8 . 7 1 6}$ & IBI (India) & $\mathbf{4 . 2 6 0}$ \\
& JIF & $\mathbf{1 . 5 0 0}$ & SJIF (Morocco) $=\mathbf{5 . 6 6 7}$ & OAJI (USA) & $\mathbf{0 . 3 5 0}$ \\
\hline
\end{tabular}

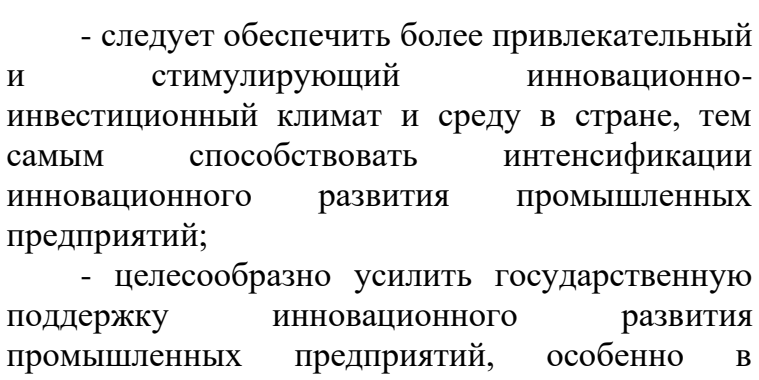

финансовых вопросах и вопросах формирования заказов на новые инновационные продукции;

- промышленным предприятиям следуем глубокое изучение прогрессивного мирового опыта по освоению и применению основных механизмов инновационного развития и в контексте международного опыта обеспечить совершенствование главных организационногоэкономических механизмов инновационного развития промышленных предприятий и т.д.

\section{References:}

1. Shumpeter, J. A. (1934). The theory of economic development.

2. Trofimov, O. V. (2011). Metodologiya formirovaniya strategii innovatsionnogo razvitiya promyshlennykh predpriyatiy $v$ usloviyakh modernizatsii ekonomiki. Diss. d-ra ekon. nauk. (p.305). Moscow.

3. Semenova, A. A. (2005). Sozdaniye natsional'noy sistemy upravleniya innovatsionnoy deyatel'nost'yu: teoriya $i$ metodologiya. Diss. d-ra ekon. nauk. (p.312). Moscow.

4. Druker, P. F. (2007). Biznes i innovatsii. (p.432). Moscow: «Vil'yams».

5. Krasnoperova, T. Y. (2013). Natsional'naya innovatsionnaya sistema: struktura, rol' finansovoy sostavlyayushchey. Nauchnotekhnicheskiy vestnik informatsionnykh tekhnologiy, mekhaniki i optiki, №6(88), pp.152156.

6. Feygin, G. F. (2009). Zakonomernosti globalizatsii i razvitiye natsional'nykh ekonomik. Diss. d-ra ekon. nauk. (p.465). Sankt-Peterburg.

7. Mel'nikov, R. M. (2008). Teoriya i mekhanizm regulirovaniya proportsiy prostranstvennogo razvitiya natsional'noy ekonomiki. Diss. d-ra ekon. nauk. (p.332). Moskva.

8. Poretskova, K. V. (2014). Formirovaniye innovatsionnoy strategii promyshlennogo predpriyatiya. Diss. kand. ekon. nauk. (p.131). Saratov.

9. Varlamov, S. A. (2014). Strategicheskiye innovatsii kak element klyuchevykh faktorov uspekha organizatsii. Ekonomika i upravleniye, №1(110), p.69-72.

10. Gubernatorov, A. M. (2015). Metodologiya $i$ organizatsiya upravleniya innovatsionnym razvitiyem otrasli. Diss. d-ra ekon. nauk. (p.342). Moscow.

11. (n.d.). Kontseptsiya razvitiya «Azerbaydzhan 2020: vzglyad v budushcheye», 41p. Retrieved 2019 , from https://president.az/files/future_ru.pdf

12. (2014). Gosudarstvennaya Programma po razvitiyu promyshlennosti $v$ Azerbaydzhanskoy Respublike na 2015-2020 gody. Utverzhdeno Rasporyazheniyem Prezidenta Azerbaydzhanskoy Respubliki ot 26 dekabrya 2014 goda, № 964, 31 p. 